\title{
A method to assess national metal criticality: the environment as a foremost measurement
}

\author{
Disna Eheliyagoda (1) ${ }^{1}$, Xianlai Zeng (1) ${ }^{1} \&$ Jinhui Li (iD ${ }^{1 凶}$
}

Ever-increasing mineral demand inspires nations to inspect the metal criticality situation that would be an indispensable path to ensure supply security in a foreseeable future. A diverse range of methods has been used to analyze the criticality; however, except a few, their applicability is questionable due to varying results. This article presents and discusses an advanced method to measure the degree of national criticality of metals conjoining both previously noted and pioneer indicators while considering China as the sample at the necessary point. The formulated methodology consists of a three-dimensional framework: supply risk, environmental risk, and supply restriction risk. The risk score of each indicator under each dimension is calculated through a specifically designed methodology. The risk score range is interpreted to a general 0-100 scale. The final risk score of each dimension is determined by averaging the total indicator risk score of that dimension. The developed criticality method is applicable for countries, which take part in the mineral production. The environmental-risk assessment is performed for 56-62 countries in reference to copper and aluminum production. Further discussion in relation to the country-specific criticality is decentralized observing the risk severity of indicators under two succinct approaches: singlemetal approach and multiple-metal approach. The obtained results associated with China demonstrate that substantial criticalities can be aggregated in supply restriction and environmental sides regarding copper and aluminum, respectively. However, the environmentalrisk assessment conducted for various nations in the world shows a very low risk status except the China's situation. Although, such indicator quantifications in the proposed method are transparent, robust, reliable, and flexible to encounter medium-term perspectives, the conducted assessment is relatively static since the evaluation is almost based on the year 2015 statistics and information. Nevertheless, the created methodology will be advantageous as a decision-making tool to implement productive national strategies and policies to achieve resource sustainability. Here, a national government can address certain issues related to the metal production by distinghushing indicator values. A government can also determine what optimizations would strategically profitable in short and medium terms such as recycling, substitutes, and imports.

\footnotetext{
${ }^{1}$ State Key Joint Laboratory of Environment Simulation and Pollution Control, School of Environment, Tsinghua University, 100084 Beijing, China.

凶email: jinhui@tsinghua.edu.cn
} 


\section{Introduction}

M etals have played a focal role in human development since historical ages such as "bronze age" and "iron age", which propagated after the "neolithic" period and they are interconnected to the modern society as a condition sine qua non (Mudd and Jowitt, 2014; Nassar et al., 2015a). Minerals as far as fossil fuels convey similar geopolitical and environmental obstacles; likewise, a considerable account of metals is normally termed as "critical" due to broad consumption, unstable supply, and source restriction to several nations (Vidal et al., 2013). The physical consumption of metals is relatively higher; thus they have become an obligatory feature to drive national economies in countries (Eheliyagoda et al., 2019a; Eheliyagoda et al., 2019b) especially with high-production capacity. It is obvious that the metal demand is inevitably increasing due to growing economies and transferring into complex lifestyles; however, it is questionable how the demand would be satisfied despite the facts such as spatial discrepancies in metal abundance, issues in achieving economically mineable ore reservoirs, and disparities in national level technological usage (Graedel et al., 2015). If such resources are not sufficient to fulfill a country's demand, the national strategic plan or the elasticity of prospective use of techniques may be collapsed (Nassar et al., 2012). Thus, it is vital to evaluate the criticality of metals in a country to ensure whether the locality will be able to secure the mineral supply situation in a foreseeable future.

The criticality of a mineral has been defined through various dimensions according to scholars' view; therefore, it is difficult to note a standard definition regarding the metal criticality. The United States National Research Council has stated an element criticality as "the risk that supplies of the element might not be routinely available together with an assessment of the impact of such a restriction on the evaluating organization" (Nassar et al., 2012); nevertheless, a national level criticality assessment requires a robust status-quo analysis with multiple indicators to address both economic threats and environmental implications (Kolotzek et al., 2018). We cautiously examined a number of criticality studies published (British Geological Survey, 2015; Calvo et al., 2018; Ciacci et al., 2016; Daw, 2017; Dewulf et al., 2016; European Commission, 2010; European Commission, 2017; Fang et al., 2018; Glöser-Chahoud et al., 2016; Graedel et al., 2012; Hatayama and Tahara, 2015; Nassar et al., 2012; Öko-Institut, 2009; Rosenau-Tornow et al., 2009; United States National Research Council, 2008; Yuan et al., 2019; Zepf et al., 2014); however, some drawback could be observed in adopting these methods for each metal at the national level. Particularly, certain indicators presented in some methodologies are a challenge to determine since data acquisition obstacles (Schrijvers et al., 2020). Overall, the supply risk of elements has been prioritized in many assessments (British Geological Survey, 2015; European Commission, 2010; Glöser-Chahoud et al., 2016; Rosenau-Tornow et al., 2009; United States National Research Council, 2008).

Previously, four studies on metal criticality have endeavored to present their methods comparatively using interesting angles beyond the supply risk. The first one is the US National Research Council assessment that has proposed a relationship between two parmeters such as supply risk and impact of supply distruption while applying several metrics for each parameter in the metal criticality determination (United States National Research Council, 2008). The second and third evaluations established by the European Commisison, is a two-axis notion regarding the supply risk that consists of three parameters and the possibile economic impact of supply restriction (Dewulf et al., 2016; European Commission, 2010; European Commission, 2017). Here, an environmental-risk evaluation has been separately included. A comprehensive indicator approach has recently developed in view of a three-dimensional scheme: supply risk, environmental implications, and vulnerability to supply restriction for three organizational levels such as globe, country, and corporate by Graedel's team at Yale University that is known as a more transparent and robust methodology among up to date published criticality studies (Graedel et al., 2012).

The environmental-risk analysis should be an important reflection in the national metal criticality determination since the metal processing phases such as mining, refining, and smelting are highly vulnerable to severe environmental impacts such as global climate change. One study on raw material criticality has aimed to assess the environmental risk from a life cycle viewpoint (Knoeri et al., 2013). Many criticality studies have limitedly highlighted the arisen environmental burden due to industrial metal processing activities; nevertheless, we believe the environmental cost accelerates the economic risk in a country since industries should spend an extra expense on nature protection if they fail to achieve environmental standards. Thus, this study attempts to introduce a better methodology to determine the national level criticality of metals in view of three dimensions, such as supply risk, environmental risk, and supply restriction risk with renewed indicators, and the combination of some indicators published in various journal articles and reliable reports. Considering the nation-wide contribution of metal production and processing, two succinct approaches are also proposed to interpret economic and environmental menace.

In the current assessment, China is used as the sample country due to two main reasons: 1 . China is a leading producer and consumer of an extensive number of metals in the periodic table, 2. a reliable and vigorous method to evaluate the criticality is still not developed considering the country's metal use status. On the other hand, the prevailing detail on raw material criticality in China is insufficient, chaotic, and difficult to access (Erdmann and Graedel, 2011). At the initial stage, copper and aluminum are regarded as instances to implement the new methodology since their extensive consumption in many industries. Although China is applied as the example country, an environmental-risk assessment approximately demonstrating all nations who invove in copper and aluminum production is also included in this national criticality evaluation. The data inadequacy is the major barrier in developing such mineral study methodologies; however, we believe that the constructed method can be adopted to assess the criticality of a wide range of metals more transparently.

\section{Methods}

The method was developed for a national level analysis considering temporal factors related to the metal criticality and in view of the medium-term perspective (i.e., until 2030). This methodology is applicable for all mineral producing countries. For examples, copper mine and aluminum smelter produced countries in 2015 are depicted in the Supplementary Information, Tables S6 and S7, respectively.

The overall framework of the study is visualized in Fig. 1. As the first part of the research, the identification and classification of indicators were performed through an extensive literature review. With the support of published articles, 18 indicators were suggested under a good balance of both quantitative and qualitative aspects covering geological, economic, environmental, technical, social, political, policy, and regulatory constraints (Graedel et al., 2012; Harper et al., 2015a; Harper et al., 2015b; Nassar et al., 2015b; Nuss et al., 2014; Panousi et al., 2016). Since the criticality method should reflect as a three-demensional framework: supply risk, environmental risk, and supply restriction risk, indicators were proposed after understanding requirements 


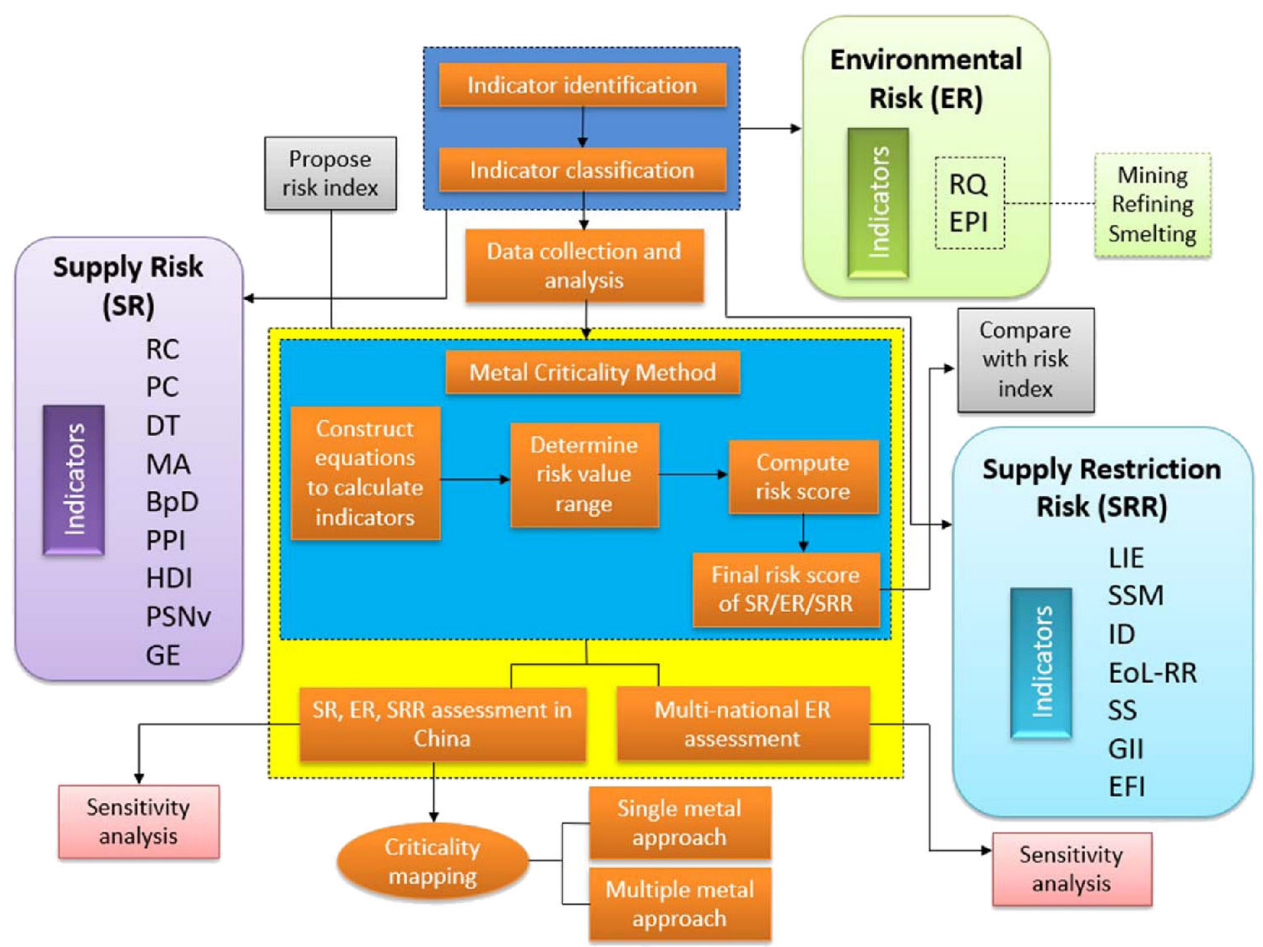

Fig. 1 An overall illustration of the research framework. The detailed flow shows steps beyond the criticality method development.

\begin{tabular}{|c|c|c|c|c|c|c|}
\hline & & & \\
\hline & & & & & Criticality Indicators & \\
\hline & & & No. & Supply Risk (SR) & Environmental Risk (ER) & Supply Restriction Risk (SRR) \\
\hline \multicolumn{3}{|c|}{ Risk Index } & 1 & Reserve Concentration (RC) & Regulatory Quality (RQ) & $\begin{array}{l}\text { Local Importance in Economy } \\
\text { (LIE) }\end{array}$ \\
\hline Risk Intensity & Score Range & Color & 2 & $\begin{array}{l}\text { Production Concentration } \\
\text { (PC) }\end{array}$ & $\begin{array}{l}\text { Environmental Performance } \\
\text { Index (EPI) }\end{array}$ & $\begin{array}{l}\text { Self Sufficiency of Mineral } \\
\text { (SSM) }\end{array}$ \\
\hline Very Low Risk & $0-10$ & & 3 & Depletion Time (DT) & & Import Dependency (ID) \\
\hline Low Risk & $10-30$ & & 4 & Mineral Adequacy (MA) & & $\begin{array}{l}\text { End-of-Life Recycling Rate (EoL- } \\
\text { RR) }\end{array}$ \\
\hline Moderate Risk & $30-50$ & & \multirow[t]{2}{*}{5} & \multirow{2}{*}{$\begin{array}{l}\text { By-product Dependency } \\
\text { (BpD) }\end{array}$} & & \multirow[t]{2}{*}{ Substitutability (SS) } \\
\hline \multirow{2}{*}{ Substantial Risk } & \multirow[t]{2}{*}{$50-70$} & & & & & \\
\hline & & & 6 & Policy Perception Index (PPI) & & Global Innovation Index (GII) \\
\hline High Risk & $70-90$ & & 7 & Human Development Index & & \multirow[t]{2}{*}{ Economic Freedom Index (EFI) } \\
\hline Very High Risk & $90-100$ & & & (HDI) & & \\
\hline & & & 8 & $\begin{array}{l}\text { Political Stability and Non- } \\
\text { violence (PSNv) }\end{array}$ & & \\
\hline & & & 9 & $\begin{array}{l}\text { Government Effectiveness } \\
\text { (GE) }\end{array}$ & & \\
\hline
\end{tabular}

Fig. 2 A summary of the risk index and criticality indicators in the study. The indicators to measure SR, ER, and SRR are proposed not only conducting a comprehensive investigation of previously published papers but novel indicators are also introduced to develop the methodology. The risk score determination routes of each indicator are prudently described in the Supplementary information.

of each dimension thoroughly. A list of suggested indicators under each dimension is shown in Fig. 2. All required datasets obtained to quantify indicators are publicly available and single or multiple sources can be used for calculations. The criticality assessment is based on 2015 data sources; however, 2016-2018 statistics were also adopted to determine a few indicators due to some issues arisen in the 2015 data usage. The unavailability of required data may influence on the final result of the assessment, but in some cases, certain variables can be removed from the methodology, which are described in the Supplementary Information. A risk index (see Fig. 2) with a common $0-100$ score scale was used to precisely present the risk intensity of each indicator under each dimension. This index was also proposed based on the scientific literature (British Geological Survey, 2015; European Commission, 2010; European Commission, 2017; Graedel et al., 2012).

After completing the prior necessitates, the national criticality method for metals was constructed. It is essential to quantify indicators to figure out a potential risk score. Therefore, equations were developed to quantify all suggested indicators as the first step. A risk value range between very low and very high risk was introduced for each indicator (see Table S1 in the Supplementary Information for an example) as a supporting method to calculating the risk score. The assessment method for each indicator is 
distinctive and each result was finalized to a risk vlaue as the second step. Then, the obtained risk value of each indicator was converted to the risk score. All these steps are fulfilled with the help of scientific publications. The valuation methods until the risk score determination of indicators including the used scientific literature are summarized in Table 1 . A further informative discussion is presented in the Supplementary Information intensely explaining the calculations of corresponding indicators of each dimension. Combining all indicators considered in each dimension of the criticality, a common equation was used to uncover the average value of supply risk, environmental risk, and supply restriction risk that is presented below.

$$
\mathrm{SR} / \mathrm{ER} / \mathrm{SRR}=\sum \frac{\text { Risk score of concerned indicators }}{\text { Number of indicators }}
$$

The resultant average value of supply risk (SR) or environmental risk (ER) or supply restriction risk (SRR) is equal to the final risk score of each dimension. By comparing the final score with the risk index, the risk severity/intensity of indicators/ dimensions can be determined.

In this study, we applied the developed method for assessing China's copper and aluminum criticality status to demonstrate how this method can be performed at the national scale. Although all three-dimensional assessment was performed to one country, a mult-national ER assessment was also conducted for a broad range of countries. A sensitivity analysis to determine the possible uncertainties, which can be occurred at $+5 \%$ and $-5 \%$ of the calculated value of each indicator and dimension is comprised in the criticality evaluations. Thus, the calculated value is denoted as " $\mathrm{C}$ " and results of the sensitivity analysis is presented as "W". Nevertheless, the maximium uncertainty is represented as 100 while presenting 0 as the minimum due to the proposed risk score range is in $0-100$. Then, we introduced two approaches: singlemetal approach and multiple-metal approach to mapping the criticality for a well-understandable interpretation while using China as the example. In the single-metal approach, we equally weighted SR, ER, and SRR by inspecting the degree of risk to interpret net resultant as a whole. For that purpose, we selected indicators at the range between substantial risk and very high risk as prioritized indicators in each dimension to implement the approach. Furthermore, we used overall risk scores resulted in SR, ER, and SRR to model the multiple-metal approach. Here, SR and SRR are interpreted as subsets of economic risk. As in the singlemetal approach, we selected the substantial risk score and over this intensity range to interpret SR, ER, and SRR for each metal in the multiple-metal approach. Finally, comprehensive sensitivity analyses for all indicators in ER and selected indicators in SR and SRR were conducted regarding copper and/or aluminum at country levels to present the degree of accuracy of the study. The performed method of the sensitivity analysis is described in the Supplementary Information.

Since indicators represent relevant constraints to reflect SR, ER, and SRR of a certain metal, a further discussion on these constraints and reasons for why these indicators are selected to assess the metal criticality with appropriate literature evidences is articulated below.

Supply risk (SR). Supply risk (SR) of a metal was calculated in view of the primary resource availability and production of a country. The national SR consists of nine indicators as shown in Fig. 2. The first five indicators are enclosed the geological, economic, and technical nature of a country while the last four indicators are based on social, political, and policy status of the same location.

The five indicators representing geological, economic, and technical scales are featured to demonstrate the parameters, such as primary metal availability, abundance, and production capacity that would be more influential to justify SR in a given country. It is important to address these parameters of mineral interest in measuring SR since the feasibility of acquiring primary sources is the main challenge at a national system. The geological reserve accumulation is much subjected to mineral supply due to its economic potential in mine extraction at the time of determination (Graedel and Nassar, 2013; United States Geological Survey, 2019) while supporting to fulfill the demand of the host country in a substantial proportion only through a marginal or zero dependence on others. In this case, SR becomes a lower stage since the existing primary resources play a pivotal role in demand reduction. Likewise reserve concentration, high-production concentration is advantageous in decreasing the supply risk. Not only a country obtains a monopoly or oligopoly in global mineral availability and/or production but these indicators are also concentrated to several countries that can considerably affect SR fluctuations. The reserve depletion period is in local importance to detect how long a country will likely balance the supply and demand for a certain metal. In the medium-term perspective, reserves would be a good relative measurement in determining the depletion time (Graedel et al., 2012; Nassar et al., 2012). Comprising entitlements to improve the national level resource production such as overseas mine entitlements to increase the primary production capacity is an efficient measure in stabilizing the supply (Hatayama and Tahara, 2015). Mineral adequacy (MA) is an indicator introduced to interpret such necessitates. Although a few commonly used metals such as aluminum, iron, and copper are typically abundant in the earth's crust (Mookherjee and Panigrahi, 1994), a range of metals like gallium, germanium, and indium faces challenges in supply since they are combined with "carrier/major metals" in relatively minor quantities as "by-products" (European Commission, 2010; Nassar et al., 2015a; Schwarz-Schampera and Herzig, 2002). In such cases, the by-production is driven as a crucial and fragile concern to altering circumstances towards supply stability.

The social, political, and policy configuration of a particular country can either obstruct or accelerate the development of economic minerals. The governance and social approaches are interlinked with mining operations since metal supply can constrain according to these jurisdictions. Generally, negative socio-economic and ecological consequences due to mining activities are led to cultivate rigid government policies/regulations and societal objections. Policy Perception Index (PPI), a composite index that measures the mining policy desirability in jurisdictions at the country level (Stedman and Green, 2017; Wilson, 2018) is used to quantify the policy scale in SR assessment. One indicator, Human Development Index (HDI), is a synopsized calculation to presume the country-specific average performance in the human development process (United Nations Development Program, 2010; United Nations Development Program, 2018), is also employed to quantify social attitudes in this valuation. Out of six composite governance dimensions of the Worldwide Governance Indicators (WGI) (World Bank, 2018), two [i.e., Political Stability and Non-violence (PSNv) and Government Effectiveness (GE)] are represented in the study to quantify the political scale in SR determination. Whilst the political instability of a nation may lead to a higher risk of metal supply (Graedel et al., 2012), the attractive government efficacy would help to develop prolific mining/ industrial policies and inspire the local and foreign business investment.

Environmental risk (ER). The environmental degradation is probably connected to the metal extraction and processing 


\section{Table 1 A summary of assessing the indicators' criticalities. Further detail is elaborated in the Supplementary Information.}

\section{Indicator}

Reserve concentration (RC)

Production concentration (PC)

Depletion time (DT)

Mineral adequacy (MA)

By-product dependency (BpD)

Policy Perception Index (PPI)

Human Development Index (HDI)

Political stability and non-violence (PSNv)

Government effectiveness (GE)

Regulatory quality (RQ)

Environmental Performance Index (EPI)

Local importance in economy (LIE)

Self-sufficiency of mineral (SSM)

Import dependency (ID)

End-of-life recycling rate (EoL-RR)

Substitutability (SS)

Global Innovation Index (GII)

Economic Freedom Index (EFI)

\section{Method description}

A country's reserve quantity of the mineral should be interpreted as a global percentage first. The data at a country level can be obtained from USGS. A certain risk value range was proposed using several publications (British Geological Survey, 2015; Graedel et al., 2012; Zepf et al., 2014) to calculate the indicator's risk score. Observing the appropriate risk value range in which the percentage could be included (see Table S1), the percentage value was converted to a score for the study's object. The Eq. S1 was adopted to find the converted score. Finally, risk score of the metal was calculated using Eq. S2.

A country's primary production of the metal should be interpreted as a global percentage first. The risk value range and risk score determination method are similar to the RC section. The country-specific production detail can be extracted not only from USGS but from local government reports and web sites also.

The static DT can be estimated as a ratio of the quantity of reserve to mine production in a given country. Here, a risk value range (see Table S2) was proposed to determine the risk score of the metal using Graedel et al. (2012) and Zepf et al. (2014). The risk score calculation method is also similar to the RC section.

The indicator was determined using three parameters: national mine production, overseas purchased mine production, and primary demand of the mineral. Since overseas mine acquisition detail of a nation is not publicly available, it is necessary to consult responsible authorities and associations to get access for these secured data. The annual primary demand of a metal and then, the MA can be calculated from Eqs. S4 and S3, respectively. The proposed risk value range can be obtrained from Table S3 and the risk score determination is similar to the RC section.

The $\mathrm{BpD}$ of a metal was calculated as a ratio of the amount of by-production to total mine production. In most cases, by-production data is not available. To solve this, the carrier/host metal production can be substituted to calculations (Graedel et al., 2012). The proposed risk value range for BpD is interpreted in Table S4. Here, the risk score can be simply calculated by multiplying the obtained risk value from 100 .

The country-specific statistics on the PPI, HDI, PSNv, and GE can be obtained from reports of the Fraser Institute annual survey of mining companies, UNDP, and Worldwide Governance Indicators, respectively. For these four indicators, a similar methodology was used to detect the risk value of each indicator and its range is depicted in Table S5. It should be noted that PPI is related only to the mining component while other three indicators can be used with mining or refining or smelting components. The risk value of each indicator was evaluated using Eq. S5. In the $\mathrm{HDI}$, the score is presented as a ratio that can be simply transformed to 100 by multiplying the published value from 100. The calculated risk value of each indicator was converted to a score for the study's purpose. The converted score and risk score were determined using Eqs. S1 and S2 as in the RC section.

As the first step, the RQ and EPI scores published by World Bank, (2018) and Hsu et al. (2016) for each jurisdiction were converted for the study's purpose by subtracting the RQ/EPI score from 100. To obtain a RQ/EPI score for a metal, the converted RQ/EPI scores were weight averaged by the country's annual mining production, refining production, and smelting production, respectively (Graedel et al., 2012). The resulted country-specific fractional contribution, then interpreted as a percentage from total global fractional contribution to determine the intensity of the risk. This percentage is equal to the RQ/EPI risk score of the nation (see Tables S6 and S7 to understand the process).

The LIE determination is based on the method used for "National Economic Importance" indicator detection mentioned in Graedel et al. (2012) with a few modifications. Prior to the LIE calculation, the apparent consumption of a metal should be determined. It can be computed using Eq. S6. Then, the risk value of the LIE indicator can be obtained from Eq. S7. Price and GDP data are recorded in USGS and World Economic Outlook. The used risk value range for the LIE risk score detection is shown in Table S8.

The SSM prioritizes the own metal production strength of a country without imports and its risk value can be calculated from Eq. S8. Required parameters are generally recorded in USGS, government reports, and related web sites. The adopted risk value range for the risk score detection is depicted in Table S9. The risk score valuation method is similar to the RC section.

The ID of a metal was calculated as a ratio of imports to the total supply. The total supply of a metal in a given country can be estimated from Eq. S9. The metal import and supply data can be extracted from USGS, government reports, and related web sites, but in data challenge occasions, some variables can be omitted appropriately. The risk value range for the ID is shown in Table S10. The risk score can be simply investigated by multiplying the obtained risk value from 100

The EoL recycling rates of metals can be found from published literature and responsible international reports (i.e., UNEP). The rest of the method is equal to the RC section. The risk value range applied for this indicator is displayed in Table S11.

Three variables such as price ratio, consumption status, and relative abundance were proposed in reference to some publications (British Geological Survey, 2015; Graedel et al., 2012; USGS) to determine the SS of a metal. Here, a suitable primary substitute to the metal is suggested first after a comprehensive literature review. An example on the primary substitute and end-use share selection is presented in Table S12. A simple approach to calculate the price ratio [price of the substitute/price of the reviewing metal] was adopted that is also discussed in Graedel et al. (2012). The commodity and metal price data can be obtained from London Metal Exchange and USGS. The adopted risk value range for the price ratio is shown in Table S13. The risk score was simply evaluated by multiplying the risk value from 100. In the consumption status, the risk was scored as 0 if the substitute was used in past or/and it is currently in use. If there is notable effort to manufacture a feasible substitute, the risk score was offered as 50. The relative abundance was defined as the earth's crust abundance that can be extracted from published papers, books, and reports. The risk value range applied for the relative abundance variable is depicted in Table S14. The risk score determination method of the variable is equal to the RC section. Finally, the resulted three variables' total risk score for each substitute was averaged and the overall substitutability risk score of the original metal was calculated as a weight averaged value over the end-use sectors using Eq. S10. The statistics on the GIl of countries can be obtained from Cornell University, INSEAD and WIPO (2015). When utilizing the risk score, the available Gll score was simply converted by subtracting it from 100 in order to obtain a lower risk score for a greater innovation.

The country level EFI was extracted from Miller and Kim (2016) to measure its impact at a supply restriction. As in the RC section, the converted score and risk score were calculated. The EFI risk value range is presented in Table S15. 
activities occurring significant negative impacts in diverse ecosystems such as eco-toxicity, greenhouse gas emissions to three natural spheres (i.e., atmosphere, hydrosphere, lithosphere) and high-energy carriage (Reller, 2011; Zepf et al., 2014). The environmental country risk is sufficient to qualify a mineral for criticality when it is aggregated with economic risk (European Commission, 2010; Frenzel et al., 2017) that is associated with supply risk (SR) and supply restriction risk (SRR) of the current study. Moreover, preserving the environmental threshold by responsible industries and authorities is important in mining and processing operations since it is vulnerable to assemble the national economic risk in a substantial proportion pushing them to disburse an extra amount on nature safeguard. For an appropriate environmental justification, a WGI indicator, Regulatory Quality (RQ) (World Bank, 2018), as well as Environmental Performance Index (EPI) (Hsu et al., 2016) published by Yale University and Columbia University in collaboration with the World Economic Forum are used to implement the ER dimension in the criticality method.

Regulations are indispensable to analyze the environmental commitment towards industrial metal congregation activities at the country level. Especially, mining, refining, and smelting activities are more susceptible to releasing a significant amount of emissions to three spheres. The metal toxicity would also act as a negative agent for human and ecosystem health when industrial metal operations are accountable to incline emissions. Thus, the government regulatory framework should have a key role in strengthening environmental safeguard and good governance that is also combined with economic security. The EPI ranks the nations' efficacy in solving prioritized environmental issues under two broad categories: protection of human health and conservation of ecosystems. Furthermore, EPI scores are compiled to evaluate the national performance in nine issue areas consisted of $>20$ indicators. While measuring the country's proximity to compromise internationally conferred targets or, in the absenteeism of covenant targets, EPI also compares each nation's ability towards environmental conservancy (Hsu et al., 2016).

Supply restriction risk (SRR). Supply restriction risk (SRR) of a metal was estimated considering the global supply shortage and the importance of the national economic development. It is rather necessary to understand how much risk a country to be tackled due to a global supply restriction. Any country is not selfsufficient to overcome own commodity needs itself. This obstacle range fluctuates depending on several factors; however, the influential weights of these factors are different for each country. As in Fig. 2, SRR encompasses seven indicators in this study. It is necessary to assess the end-use application of each metal in the evaluation of SRR that varies depending on the degree of national importance and the substitutability of the metal (DeYoung et al., 2006; United States National Research Council, 2008). Countryspecific requirements to meet certain circumstances referring to self-production capacity, import reliance, economic resilience, and knowledge \& technology development also consist at the indicator list of SRR.

Metals would contribute at great importance for national economic development in a country (Graedel et al., 2012; Hatayama and Tahara, 2015; Nassar et al., 2012). Large quantities of metal production, particularly for sourcing-nations, are noticeably responsible for the national gross domestic product (GDP) inflation. Thus, this necessity is interpreted as an indicator under SRR. The self-ability to produce mineral resources is essential to meet a supply shortage. Therefore, Self-Sufficiency of Mineral (SSM) indicator tries to measure a country's capacity to overcome a global supply restriction. Dependency on imports is one of the major threats, a country should address if a global supply shortage of minerals occurs. The high susceptibility for importations indeed leads to many issues in the case of a supply interruption. Recycling can apply at various stages in the anthropogenic life cycle of mineral production and manufacturing while declining SRR. The end-of-life (EoL) product recycling is strongly encouraged in today's world since its substantial benefits, i.e., cost-effectiveness, technical feasibility, energy savings in the process (Rademaker et al., 2013). A higher EoL recycling rate may perhaps lead to a decrease in primary resource demand, material substitution, and energy demand (British Geological Survey, 2015; European Commission, 2010). Substitutes are potential applications to replace one commodity to another at a supply interruption that may reduce the risk while decreasing the raw material use of the corresponding commodity (British Geological Survey, 2015; Glöser et al., 2015); however, the substitution is specifically suitable to alter potentially scarce and hard recycling critical metals (European Commission, 2010). Although some characteristics of metals are irreplaceable, a consumable substitute can be discovered with time (Graedel et al., 2012).

A more innovative country means that it inherently obtains a good capacity to find new solutions and ways to overcome a potential supply restriction; thus, these nations are less vulnerable to an SRR (Graedel et al., 2012). Economic freedom provides greater opportunities for innovation, progress, and human flourishing. It is a good measurement to evaluate the market openness for novelty that is also beneficial to overcome the impact of global supply interruption towards a country. To measure these requirements, Global Innovation Index (GII) (Cornell University, INSEAD and WIPO, 2015) and Economic Freedom Index (EFI) (Miller and Kim, 2016) are utilized as indicators in the SRR determination.

\section{Results}

Supply risk (SR) in China. The SR assessment (Fig. 3) demonstrates that copper and aluminum in China are still not in a noticeable risky position. The analyses also yield a medium supply risk score of 46 for copper and a low supply risk score of 18 for aluminum. Copper shows a different picture than aluminum that convinces the possibility to reach a substantial risk level in the near future due to considerable risk intensities represented by a considerable number of indicators. Nevertheless, only two indicators of aluminum show a substantial risk condition in SR. Furthermore, Fig. 3 demonstrates an essential aspect in the assessment; that single metrics are able to exhibit a high level of criticality (as in the DT and MA risk scores of copper, DT and RC risk scores of aluminum) even though the overall SR estimate is moderate or marginal. The mentioned circumstance underlines the necessity of totaling a full array of influential factors rather than one or a small number of indicators in assessing the metal criticality. Aggregated uncertainties of several copper SR indicators regarding global source-nations are illustrated in the Supplementary Information, Fig. S3.

Environmental risk (ER) in China. Figure 3 shows that aluminum mining and processing operations lead to a substantial risk potential in the Chinese environment accounting for the overall risk score as 53 that impels to focus on suitable technological modifications regarding mining and smelting operations to safeguard nature. In the case of alumina production and aluminum smelter production, relevant stakeholders and authorities should be more responsible to maintain environmental standards and cleanliness. The major environmental impact of energyintensive aluminum production is the considerable emit of 


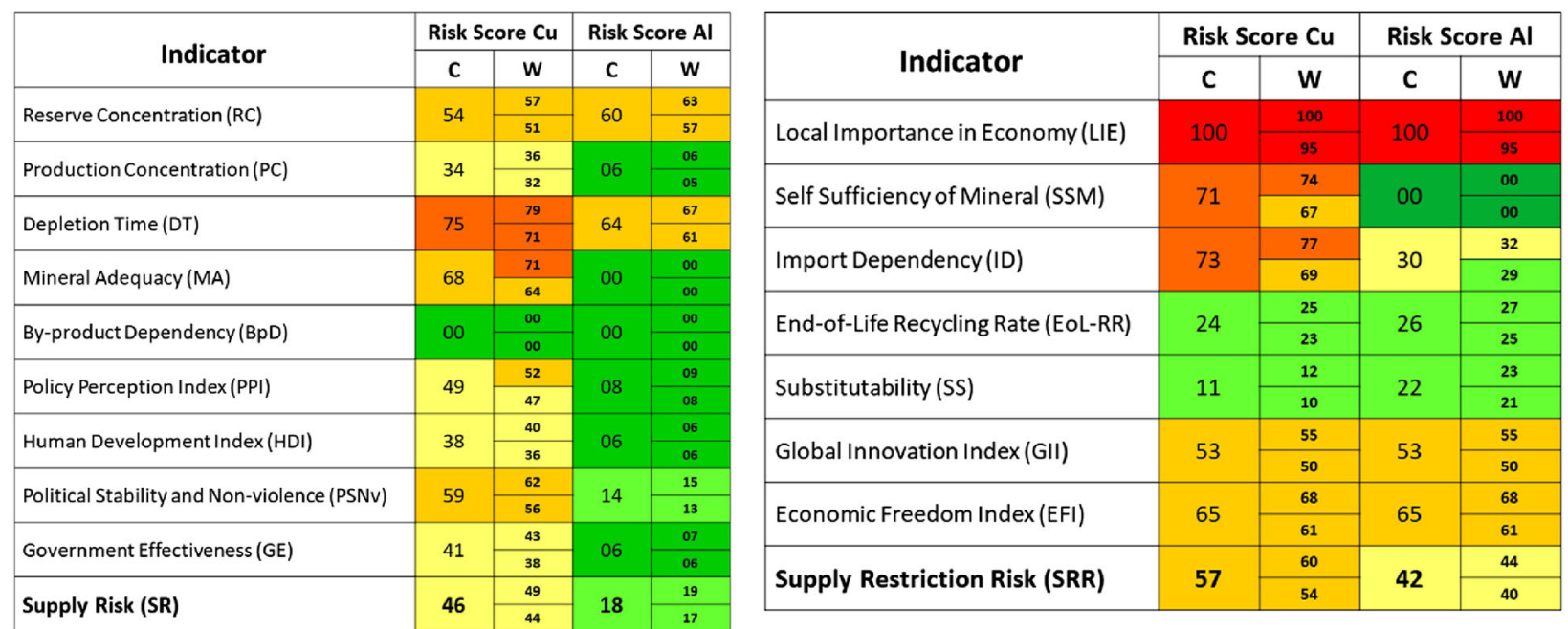

\begin{tabular}{|c|c|c|c|c|c|c|c|c|c|c|c|c|}
\hline \multirow{3}{*}{ Indicator } & \multicolumn{6}{|c|}{$\begin{array}{c}\text { Risk Score } \\
\mathrm{Cu}\end{array}$} & \multicolumn{6}{|c|}{$\begin{array}{c}\text { Risk Score } \\
\text { Al }\end{array}$} \\
\hline & \multicolumn{2}{|c|}{$\begin{array}{l}\text { Mining } \\
\text { (M) }\end{array}$} & \multicolumn{2}{|c|}{$\begin{array}{c}\text { Refining } \\
\text { (R) }\end{array}$} & \multicolumn{2}{|c|}{$\begin{array}{c}\text { Smelting } \\
\text { (S) }\end{array}$} & \multicolumn{2}{|c|}{$\begin{array}{c}\text { Mining } \\
\text { (Bauxite) } \\
\text { (Mb) }\end{array}$} & \multicolumn{2}{|c|}{$\begin{array}{l}\text { Mining } \\
\text { (Alumina) } \\
\text { (Ma) }\end{array}$} & \multicolumn{2}{|c|}{$\begin{array}{c}\text { Smelting } \\
\text { (S) }\end{array}$} \\
\hline & c & $w$ & c & w & c & w & c & $w$ & c & w & c & w \\
\hline \multirow{2}{*}{ Regulatory Quality (RQ) } & \multirow[b]{2}{*}{15} & 16 & \multirow[b]{2}{*}{49} & 51 & \multirow[b]{2}{*}{52} & 55 & \multirow[b]{2}{*}{31} & 32 & \multirow[b]{2}{*}{65} & 68 & \multirow[b]{2}{*}{66} & 69 \\
\hline & & 14 & & 46 & & 50 & & 29 & & 62 & & 63 \\
\hline \multirow{2}{*}{$\begin{array}{l}\text { Environmental Performance } \\
\text { Index (EPI) }\end{array}$} & \multirow{2}{*}{12} & 13 & \multirow{2}{*}{43} & 45 & \multirow{2}{*}{47} & 50 & \multirow{2}{*}{28} & 30 & \multirow{2}{*}{63} & 67 & \multirow{2}{*}{64} & 67 \\
\hline & & 11 & & 41 & & 45 & & 27 & & 60 & & 60 \\
\hline \multirow{3}{*}{ Environmental Risk (ER) } & \multicolumn{3}{|c|}{ c } & \multicolumn{3}{|c|}{ w } & \multicolumn{3}{|c|}{ C } & \multicolumn{3}{|c|}{ w } \\
\hline & \multirow{2}{*}{\multicolumn{3}{|c|}{36}} & \multicolumn{3}{|c|}{38} & \multirow{2}{*}{\multicolumn{3}{|c|}{53}} & \multicolumn{3}{|c|}{56} \\
\hline & & & & \multicolumn{3}{|c|}{34} & & & & \multicolumn{3}{|c|}{50} \\
\hline
\end{tabular}

Fig. 3 The criticality evaluation for copper and aluminum in China. Results of each dimension (SR, ER, and SRR) are concisely interpreted. The step by step process to ascertain the risk score of each single indicator is vigorously explained with relevant data sources and assumptions in the Supplementary Information.

greenhouse gases. Copper environmental implications account for a moderate risk scoring 36 in Chinese spheres. When copper discharges to acidic soil, it distributes copper ion traces that are toxic from micro to macro levels of biotic organizations. Copper is highly susceptible to bio-accumulate in macro biotas such as plants and animals since it does not break down in the environment (Zepf et al., 2014).

Multi-national environmental risk (ER). The ER assessments covering 62 and 56 nations who engage in copper and aluminum production are presented in Fig. 4a, b, respectively. Research results demonstrate that almost all countries except China are in a safe position regarding the risk pertaining to the environment due to production operations while showing comparatively a very low risk. Nevertheless, a few countries show small deviations regarding individual ER indicators but they are also in the low risk range. China is the sole country that can considerably aggregate environmental impacts due to the aluminum production while showing a moderate risk score in the copper production. China's premier alumina and primary smelter production has accelerated substantial issues in the environment. As the top mine producer of copper, Chile produces $>3$ times of China's production but the RQ risk related to the copper mining is lesser than that of China's score. This may occur due to the quality of regulations in Chile compared to China. Even though Peru and United States are leading producers, their environmental risk is still relatively lower. As the top producer of bauxite, Australia produces approximately $>25 \%$ of China's production but the environmental risk related to bauxite mining is lesser than that of China's corresponding indicator score. This may occur due to the quality of regulations and environmental performance in Australia compared to China. Even though some leading producers such as Brazil and India are facing some regulatory and environmental performance issues, their environmental risk is still relatively lower. However, it should be noted that the averaged ER is better than the score used for single indicators in the risk evaluation. Indicator-wise broad clarifications regarding multinational ER assessments for copper and aluminum are depicted in the Supplementary Information, Figs S1 and S2, respectively. Moreover, aggregated uncertainties of copper and aluminum ER including corresponding indicators of producing countries are shown in the Supplementary Information, Figs S4 and S5.

Supply restriction risk (SRR) in China. The study yields substantial (57) and moderate (42) risk scores for copper and aluminum in the SRR assessment, respectively (Fig. 3). It is obvious that China relies on more copper imports to fulfill the demand; however, aluminum ID risk is at a low level mainly due to China's higher primary production volume compared to other nations. Therefore, the risk pertained to the self-sufficiency of aluminum is much lower than the rest of the indicators' values. Figure 3 also demonstrates a key aspect of SRR evaluation; that no single approach is apposite to assess a full range of metal criticality since one indicator can point out an elevated criticality (as in the LIE rating for aluminum), but the overall SRR output would be finalized to moderate intensity. Aggregated uncertainties of two 


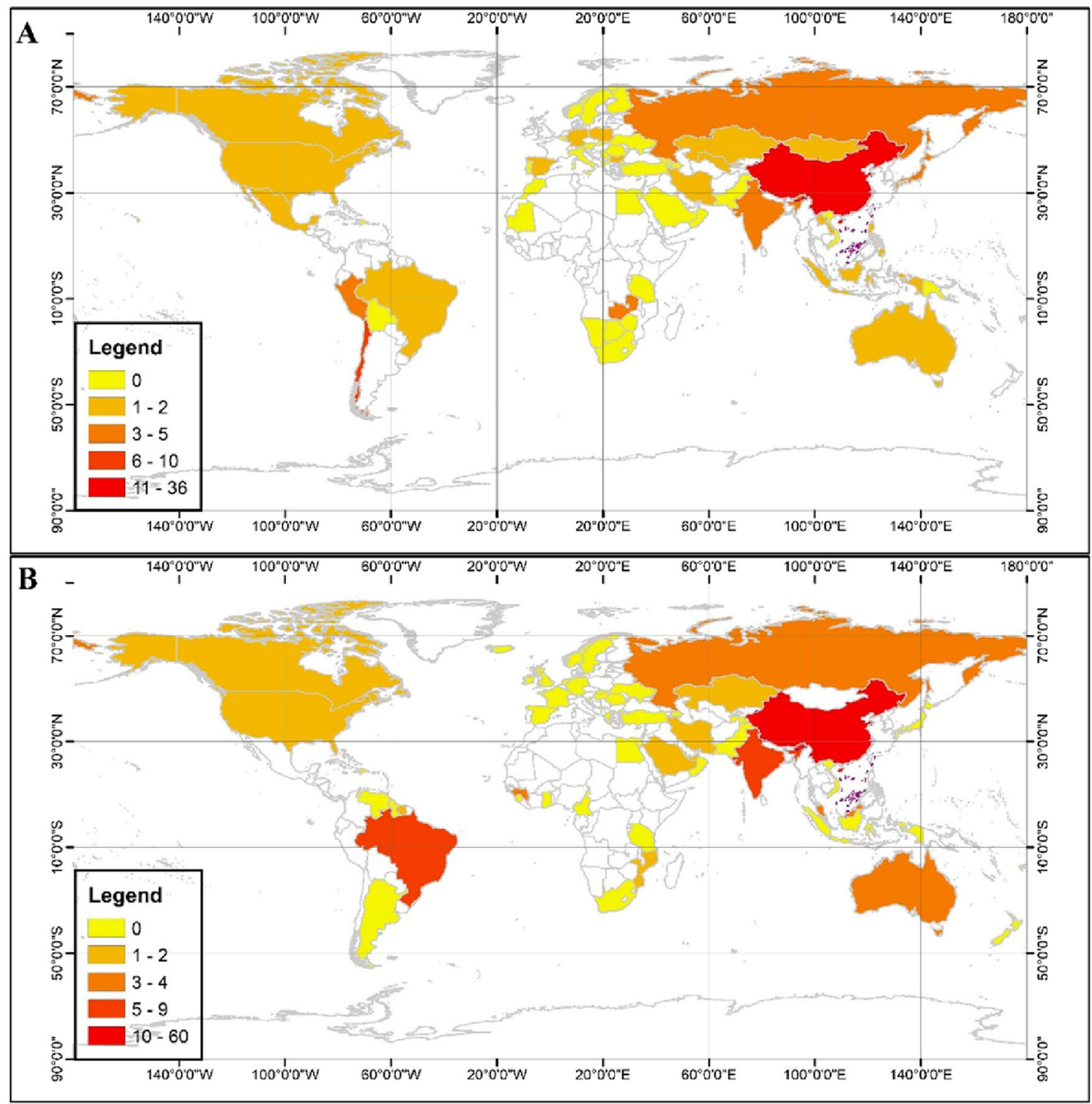

Fig. 4 Multi-national environmental-risk (ER) interpretation. a Copper. b Aluminum. These assessments are conducted for all countries, which contributed to production operations.

copper and aluminum SRR indicators (GII and EFI) for a broad range of countries are shown in the Supplementary Information, Fig. S6.

\section{Mapping the criticality in China}

Single-metal approach. National criticality assessments of copper and aluminum under the single-metal approach regarding China are presented in Fig. 5. The SR and SRR represent the magnitude of the economic risk of a nation that can be pertained due to metal criticality and these two dimensions are connected to ER according to the study's perspectives. The tendency to become more critical is not identical under each dimension of different metals and they are dependent on the risk intensity of indicators. In the case of Chinese contemporary conditions, copper is susceptible to accelerate economic risk since the majority of indicators comprised in SR and SRR demonstrates a considerable risk intensity whereas ER of copper is relatively low (Fig. 5a). In contrast, aluminum is responsible for accumulating a significant amount of negative burden in the environment; however, its economic risk is relatively at a minute quantity when viewing at the aggregation of indicators in SR and SRR as depicted in Fig. 5b.
Multiple-metal approach. China is currently the leading global producer of 23 elements (British Geological Survey, 2015) consuming a diverse range of minerals in the periodic table. The single-metal approach is rather difficult to apply for source countries, which produce and process comparatively a higher number of metals such as China, the United States, and Australia so on. The main reason for that is the complexity and chaos in the interpretation of individual indicators for each metal separately. Therefore, the multiple-metal approach is secondly introduced. The proposed criticality analysis regarding China demonstrates that copper is in a risky position at the supply restriction (SRR) whereas aluminum intensifies the environmental risk (ER) (Fig. 6). Using such a comprehensive approach, there could be observed a dissimilar level of criticality between copper and aluminum in China.

\section{Discussion}

The current study introduces a robust and flexible threedimensional methodology to assess the medium-term metal criticality (i.e., producing countries are prioritized) suggesting indicators to process the criticality evaluation. This criticality 


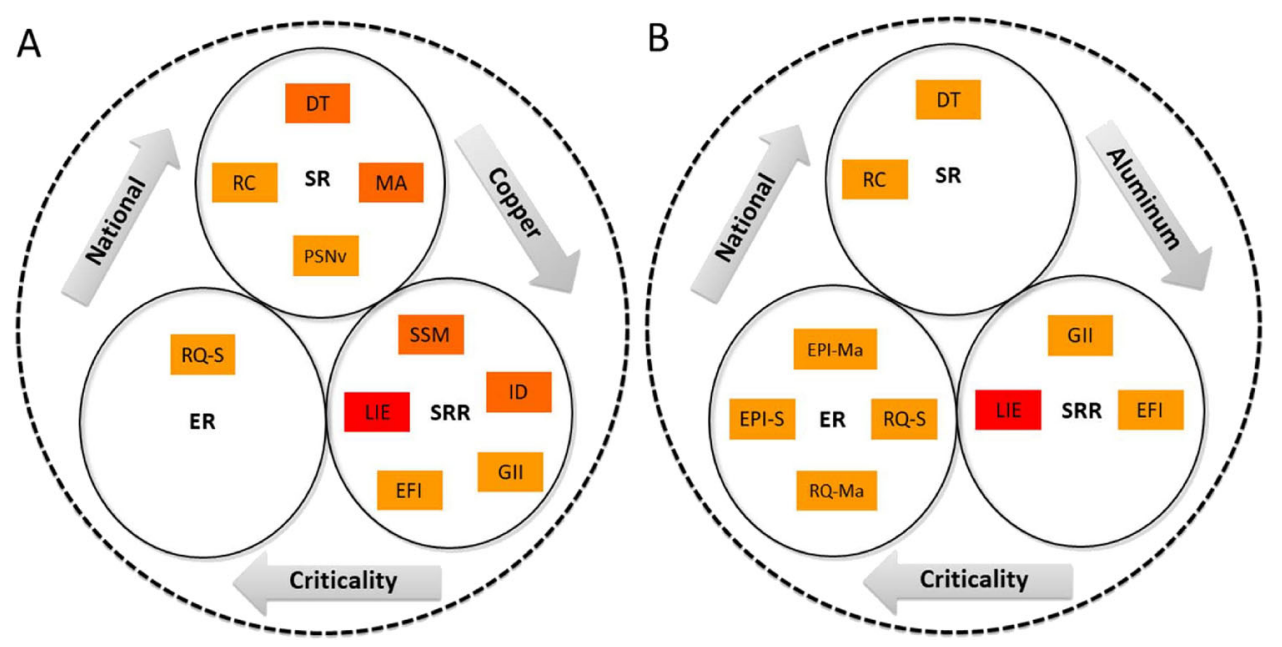

Fig. 5 National level criticality assessment using the single-metal approach. a copper. $\mathbf{b}$ aluminum. The single-metal approach is adopted to examine China's criticality situation.
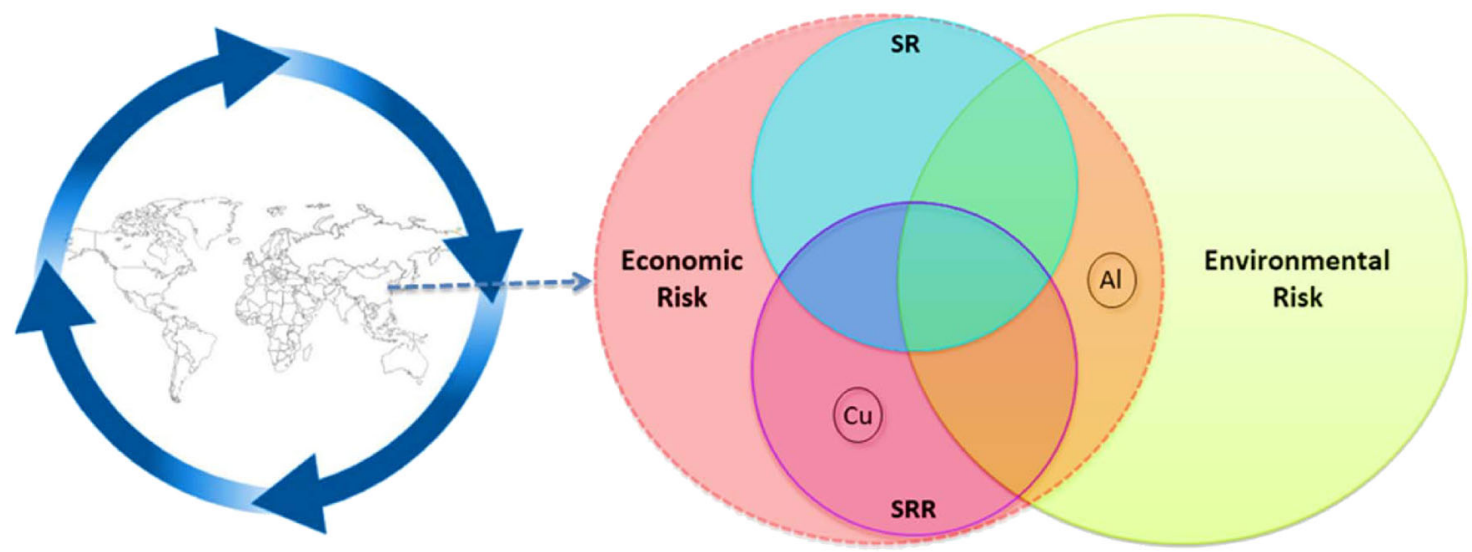

Fig. 6 National level criticality assessment using the multiple-metal approach. China's criticality status is demonstrated under the multiple-metal approach.

methodology is solely developed to analyze a national level situation taking China as the example country. The use of a few indicators is not appropriate to measure the mineral criticality as a time scale snapshot (Graedel et al., 2012); thus, multiple indicators are included to construct a precise and accurate method (Graedel et al., 2012; Graedel and Nuss, 2014; Nassar et al., 2012). Accordingly, three dimensions: supply risk (SR), environmental risk (ER), and supply restriction risk (SRR) consist of nine, two, and seven indicator numbers, respectively, representing certain constraints regarding the metal criticality. The evaluation scheme of each indicator is extensively described to proliferate the accuracy and precision of the proposed method. A comprehensive criticality assessment should include a wide range of data inputs addressing relatively whole conditions and phenomena influenced from quantitative scientific approaches to qualitative social and regulatory/policy aspects; nevertheless, there could be discrepancies in these data interpretations since some relevant data are not well defined (Graedel et al., 2012). Almost all data used in the study are obtained from publicly available reliable sources, which represent a broad variety of spectrum. Since this assessment is relatively static, indicator valuation data are based on year 2015 statistics. On the one hand, this year provides an extensive range of data inputs required to assess each indicator.
On the other hand, this annual selection may build a proximity to the current and near future scenarios. Although chosen indicators and their assessment methods can be debated, we presented this method in an immensely transparent nature to mitigate the complexity arisen due to the application of quantitative and qualitative constraints. As performed in the study, we think that averaging the whole indicator risk score is more flexible and accurate rather than the use of a single indicator value as its final score (Graedel et al., 2012; Nassar et al., 2012). Additionally, sensitivity and uncertainty analyses are developed to discuss the degree of uncertainties that can be aggregated when applying the methodical circumstances.

We believe that the criticality mapping is an indispensable aspect to analyze a nation's overall metal outlook through various angles for a better interpretation. Therefore, the national metal criticality is comprehensively demonstrated introducing two different approaches: single-metal approach and multiple-metal approach. At a national level criticality assessment, the impact of the metal processing industry on the economy and environment can be viewed through the angle of single-metal closely looking at its individual indicators. This concept can be reflected as a core fact for nations that produce and process several metals since certain industries would be able to formulate appropriate 
decisions considering highly prioritized indicators to overcome the criticality. The proper understanding of the nature of the corresponding components will raise the efficiency of the criticality evaluation (Hatayama and Tahara, 2015). It may advantageous for localities to construct resource strategies meaningfully. The single-metal approach is appropriate to use as a decisionmaking tool in resource strategic planning within the framework of "single-user" since individual metal indicators are separately addressed in this approach. Since, the single-metal approach is difficult to apply for nations, which produce comparatively a higher number of minerals, the multiple-metal approach is introduced to solve the complications in such situations. If a country produces a significant number of minerals, it can be expected that the corresponding country's economy is more dependent on mineral related attributes, i.e., exports of refined minerals, semi-fabricated, and finished products. Therefore, SR and SRR can largely contribute to accelerate the economic risk. As a consequence, we interpreted SR and SRR as subsets of economic risk in the multiple-metal approach. One of the principle drawbacks of many studies is the limited specification of environmental role at the national criticality evaluation; however, mineral processing actions noticeably deteriorate the quality of nature that would eventually aggregate unprecedented catastrophes such as atmospheric imbalance in the planet Earth (Azadi et al., 2020; Liu et al., 2012). Hence, environmental consequences may intervene to increase the economic risk in a country. Globally, there is no demarcation in the distribution of atmospheric pollutants since one country's atmospheric contamination promptly affects to the neighborhood degrading the whole planet's well-being to some extent. Thus, quantifying both economic and environmental costs as equivalent components is rather optimistic regarding mineral criticality estimations. As a result, equal weight is offered to each component (i.e., economic risk and environmental risk) in the multiple-metal approach that is the major discrepancy compared to the single-metal approach. Besides that, economic risk and environmental risk are interlinked to interpret the relevancy of these two components.

The developed method is perhaps a time scale snapshot leading results to medium-term projections; however, some indicators (for instance, Import Dependency) are short-term while changing slowly. In this study, appropriate time scale data are used in the determination of the influence of each indicator wherever obtainable. It should be noted that indicators are dynamic and willing to change over time; nevertheless, this study would be a static assessment since the basic year used to the analysis is 2015 with a few exceptions. Therefore, further studies will be required to develop a dynamic evaluation regarding metal criticality.

The aggregated risk scores presented for each indicator describe a broad scope in measuring the criticality that may be beneficial to develop national mineral strategies and suggest indispensable policy options after analyzing the criticality status of minerals. The proposed single and multiple-metal approaches will also advocate functioning resource strategies and policies observing encountered substantial criticality conditions. In other words, this assessment can apply as a decision-making tool to build consensus among different stakeholders involved in various life stages of metal usage such as consumers, producers, manufacturers, recyclers, and policymakers. This kind of a concept and action will be indeed needed to address issues arisen due to the growing potential of mineral use while developing a nation-wide vision on resource sustainability in the future.

It is obvious that anyone can excite to know what outcomes are obtained when this developed method is applied to a national level analysis of metals. We answered this question assessing copper and aluminum criticality status in China. The results demonstrate that the methodology can be positively used to distinguish the criticalities of metals at a country. A study on the metal criticality conducted by the European Commisison in 2010 reflects their results for the EU economy showing copper and aluminum are less critical. Although, both supply risk and environmental country risk are here in a low risk for both copper and aluminum, the degree of economic importance is high only for aluminum. A global scale supply risk assessment performed by the British Geological Survey in 2015 for chemical elements, which comprise of economic worth shows very low risk for copper and aluminum. One study on the Japanese context reflects moderate and low aggregated criticality scores for copper and aluminum, respectively, from five categorical indicators (Hatayama and Tahara, 2015). Another study demonstrates that the criticality vector magnitudes for copper at the global and national levels are of 32 and 44 out of 100 , respectively that can be interpreted as modest risk scores (Nassar et al., 2012). Here, the national analysis has been conducted for United States. Nevertheless, single indicator risk scores show different figures from very low to very high degree as in the present study. A study on a few metals in Australia represents substantial and moderate risk scores for copper and aluminum regarding supply risk and vulnerability to supply restriction dimensions while, respectively, showing low and very low criticalities in environmental implications for both metals (Ciacci et al., 2016). The results of last two studies are proximate to the present study output on supply risk and supply restriction; however, the environmental risk regarding these two metals in China demonstrates a considerable deviation. It should be noted that the results may differ from method to method depending on the evaluation criteria and used indicators.

\section{Conclusions}

This paper presents a new methodology to assess national metal criticality under three principal dimensions: supply risk, environmental risk, and supply restriction risk using multiple indicators, which encompass both previously identified and originally developed indicators. Totally, 18 indicators are proposed to develop the overall method, duly capturing quantitative and qualitative influential constraints regarding the mineral criticality, such as economic, technological, geological, socio-political, environmental, regulatory, and policy factors. The constructed method is applied to analyze the Chinese copper and aluminum metal criticality as an instance to validate its applicability. A multi-national environmental-risk assessment for these two metals is also conducted to present a broad array of results and optimize the quality of the research.

The research framework mainly encompasses six directions: (a) identify and classify indicators, (b) formulate the criticality method, (c) evaluate the selected metal criticality in China, (d) assess the multi-national environmental risk for selected metals, (e) map the selected metal criticality in China (f) perform sensitivity and uncertainty analyses.

The results of the study demonstrate that China's copper and aluminum are in substantial criticality status regarding the supply restriction risk and environmental risk, respectively. The introduced methodology would be useful in designing mid-term country-specific strategic schemes considering metal supply and demand scenarios.

\section{Data availability}

The supported data sources are publicly available and their citations are mentioned in references of this paper and the Supplementary Information file. Additional information regarding the study can be obtained from the corresponding author upon a reasonable request. 
Received: 18 February 2020; Accepted: 30 June 2020;

Published online: 27 July 2020

\section{References}

Azadi M, Northey SA, Ali SH, Edraki M (2020) Transparency on greenhouse gas emissions from mining to enable climate change mitigation. Nat Geosci 13:100-104

British Geological Survey (2015) Risk list 2015: an update to the supply risk index for elements or element groups that are of economic value. British Geological Survey, London, United Kingdom

Calvo G, Valero A, Valero A (2018) Thermodynamic approach to evaluate the criticality of raw materials and its application through a material flow analysis in Europe. J Ind Ecol 22(4):839-852

Ciacci L, Nuss P, Reck B, Werner T, Graedel T (2016) Metal criticality determination for Australia, the US, and the planet-comparing 2008 and 2012 results. Resources 5(4). https://doi.org/10.3390/resources5040029

Cornell University, INSEAD and WIPO (2015) The Global Innovation Index 2015: effective innovation policies for development. Fontainebleau, Ithaca and Geneva

Daw G (2017) Security of mineral resources: a new framework for quantitative assessment of criticality. Resour Policy 53:173-189

Dewulf J, Blengini GA, Pennington D, Nuss P, Nassar NT (2016) Criticality on the international scene: Quo vadis? Resour Policy 50:169-176

DeYoung JH Jr., McCartan L, Gambogi J (2006) What's been (and what will be) strategic-my metal or your paint? Proceedings of the 42nd Forum on the Geology of Industrial Minerals, Information Circular 34, North Carolina Geological Survey, Raleigh NC, United States

Eheliyagoda D, Zeng X, Wang Z, Albalghiti E, Li J (2019a) Forecasting the temporal stock generation and recycling potential of metals towards a sustainable future: the case of gallium in China. Sci Total Environ 689:332-340

Eheliyagoda D, Wei F, Shan G, Albalghiti E, Zeng X, Li J (2019b) Examining the temporal demand and sustainability of copper in China. Environ Sci Technol 53:13812-13821

Erdmann L, Graedel TE (2011) Criticality of non-fuel minerals: a review of major approaches and analyses. Environ Sci Technol 45(18):7620-7630

European Commission (2010) Report on critical raw materials for the EU. European Commission Report of the Ad-hoc Working Group on defining critical raw materials, European Commission's DG Enterprise and Industry, Brussels, Belgium

European Commission (2017) Methodology for establishing the EU list of critical raw materials. European Commission's DG Enterprise and Industry, Brussels, Belgium

Fang S, Yan W, Cao H, Song Q, Zhang Y, Sun Z (2018) Evaluation on end-of-life LEDs by understanding the criticality and recyclability for metals recycling. J Clean Prod 182:624-633

Frenzel M, Kullik J, Reuter MA, Gutzmer J (2017) Raw material 'criticality'-sense or nonsense? J Phys D: Appl Phys 50. https://doi.org/10.1088/1361-6463/ aa5b64

Glöser S, Tercero Espinoza L, Gandenberger C, Faulstich M (2015) Raw material criticality in the context of classical risk assessment. Resour Policy 44:35-46

Glöser-Chahoud S, Tercero Espinoza L, Walz R, Faulstich M (2016) Taking the step towards a more dynamic view on raw material criticality: an indicator based analysis for Germany and Japan. Resources 5(4):45

Graedel TE, Barr R, Chandler C, Chase T, Choi J, Christoffersen L, Friedlander E, Henly C, Jun C, Nassar NT, Schechner D, Warren S, Yang MY, Zhu C (2012) Methodology of metal criticality determination. Environ Sci Technol 46 (2):1063-1070

Graedel TE, Harper EM, Nassar NT, Nuss P, Reck BK (2015) Criticality of metals and metalloids. Proc Natl Acad Sci 112(14):4257-4262

Graedel TE, Nassar NT (2013) The criticality of metals: a perspective for geologists. Geol Soc 393(1):291-302

Graedel TE, Nuss P (2014) Employing considerations of criticality in product design. JOM 66(11):2360-2366

Harper EM, Kavlak G, Burmeister L, Eckelman MJ, Erbis S, Sebastian Espinoza V, Nuss P, Graedel TE (2015a) Criticality of the geological zinc, tin, and lead family. J Ind Ecol 19(4):628-644

Harper EM, Diao Z, Panousi S, Nuss P, Eckelman MJ, Graedel TE (2015b) The criticality of four nuclear energy metals. Resour Conserv Recycl 95:193-201

Hatayama H, Tahara K (2015) Criticality assessment of metals for Japan's resource strategy. Mat Trans 56(2):229-235

Hsu A et al. (2016) Global metrics for the environment: Environmental Performance Index 2016 report. Yale University, New Haven, United States
Knoeri C, Wager PA, Stamp A, Althaus HJ, Weil M (2013) Towards a dynamic assessment of raw materials criticality: linking agent-based demand-with material flow supply modelling approaches. Sci Total Environ 461-462:808-812

Kolotzek C, Helbig C, Thorenz A, Reller A, Tuma A (2018) A company-oriented model for the assessment of raw material supply risks, environmental impact and social implications. J Clean Prod 176:566-580

Liu G, Bangs CE, Müller DB (2012) Stock dynamics and emission pathways of the global aluminium cycle. Nat Clim Change 3:338-342

Miller T, Kim AB (2016) Highlights of the 2015 index of economic freedom: promoting economic opportunity and prosperity. The Heritage Foundation, Washington DC, United States

Mookherjee A, Panigrahi MK (1994) Reserve base in relation to crustal abundance of metals: another look. J Geochem Explor 51:1-9

Mudd GM, Jowitt SM (2014) A detailed assessment of global nickel resource trends and endowments. Econ Geol 109(7):1813-1841

Nassar NT, Barr R, Browning M, Diao Z, Friedlander E, Harper EM, Henly C, Kavlak G, Kwatra S, Jun C, Warren S, Yang MY, Graedel TE (2012) Criticality of the geological copper family. Environ Sci Technol 46(2):1071-1078

Nassar NT, Graedel TE, Harper EM (2015a) By-product metals are technologically essential but have problematic supply. Sci Adv 1(3):1-10

Nassar NT, Du X, Graedel TE (2015b) Criticality of the rare earth elements. J Ind Ecol 19(6):1044-1054

National Research Council (2008) Minerals, critical minerals, and the U.S. economy. United States National Research Council, National Academies Press, Washington DC

Nuss P, Harper EM, Nassar NT, Reck BK, Graedel TE (2014) Criticality of iron and its principal alloying elements. Environ Sci Technol 48(7):4171-4177

Öko-Institut eV (2009) Critical metals for future sustainable technologies and their recycling potential. Workshop on the recycling of metals: United Nations Environment Programme Panel on Sustainable Resource Management, Brussels, Belgium

Panousi S, Harper EM, Nuss P, Eckelman MJ, Hakimian A, Graedel TE (2016) Criticality of seven specialty metals. J Ind Ecol 20(4):837-853

Rademaker JH, Kleijn R, Yang Y (2013) Recycling as a strategy against rare earth element criticality: a systemic evaluation of the potential yield of $\mathrm{NdFeB}$ magnet recycling. Environ Sci Technol 47(18):10129-10136

Reller A (2011) Criticality of metal resources for functional materials used in electronics and microelectronics. Phys Status Solidi - R 5(9):309-311

Rosenau-Tornow D, Buchholz P, Riemann A, Wagner M (2009) Assessing the long-term supply risks for mineral raw materials-a combined evaluation of past and future trends. Resour Policy 34(4):161-175

Schrijvers D et al (2020) A review of methods and data to determine raw material criticality. Resour Conserv Recycl 155. https://doi.org/10.1016/j.resconrec. 2019.104617

Schwarz-Schampera U, Herzig PM (2002) Indium: geology, mineralogy, and economics. Springer Verlag, Berlin

Stedman A, Green KP (2017) Fraser Institute Annual Survey of Mining Companies, 2017. Fraser Institute, Vancouver, British Columbia, Canada

United Nations Development Programme (2010) The real wealth of nations: pathways to human development-human development report, 2010. UNDP, New York, United States

United Nations Development Programme (2018) Human Development Index (HDI), 2018. UNDP, New York, United States

United States Geological Survey (2019) Mineral commodity summaries, 2019. USGS, Washington DC, United States

Vidal O, Goffé B, Arndt N (2013) Metals for a low-carbon society. Nat Geosci 6 (11):894-896

Wilson A (2018) Global mining survey results for 2012/2013 reveals shift in Canada's top-ranked jurisdictions. http://www.fraserinstitute.org/uploadedFiles/fraser-ca/ Content/researchnews/research/articles/global-mining-survey-results-for-20122013-reveals-shift.pdf. Accessed 15 Nov 2018

World Bank (2018) The worldwide governance indicators: 2018 update. World Bank, Washington, United States

Yuan Y, Yellishetty M, Muñoz MA, Northey SA (2019) Toward a dynamic evaluation of mineral criticality: introducing the framework of criticality systems. J Ind Ecol 23(5):1264-1277

Zepf V, Simmons J, Reller A, Ashfield M, Rennie C (2014) Materials critical to the energy industry: an introduction. London, United Kingdom

\section{Acknowledgements}

The work is financially supported by the National Key R\&D Program of China (2018YFC1900101 and 2019YFC1908501) and the International Copper Association (ICA). We thank Dr. Guijuan Shan for her contribution to the project communication with ICA. 


\section{Competing interests}

The authors declare no competing interests.

\section{Additional information}

Supplementary information is available for this paper at https://doi.org/10.1057/s41599020-00537-4

Correspondence and requests for materials should be addressed to J.L.

Reprints and permission information is available at http://www.nature.com/reprints

Publisher's note Springer Nature remains neutral with regard to jurisdictional claims in published maps and institutional affiliations. (c) (i) Open Access This article is licensed under a Creative Commons Attribution 4.0 International License, which permits use, sharing, adaptation, distribution and reproduction in any medium or format, as long as you give appropriate credit to the original author(s) and the source, provide a link to the Creative Commons license, and indicate if changes were made. The images or other third party material in this article are included in the article's Creative Commons license, unless indicated otherwise in a credit line to the material. If material is not included in the article's Creative Commons license and your intended use is not permitted by statutory regulation or exceeds the permitted use, you will need to obtain permission directly from the copyright holder. To view a copy of this license, visit http://creativecommons.org/ licenses/by/4.0/.

(C) The Author(s) 2020 